\title{
An Exposition of the Connection between Limit-Periodic Potentials and Profinite Groups
}

\author{
Z. Gan * \\ Department of Mathematics, Rice University, 77005 Houston, USA
}

Dedicated to the memory of M. Sh. Birman.

\begin{abstract}
We classify the hulls of different limit-periodic potentials and show that the hull of a limit-periodic potential is a procyclic group. We describe how limit-periodic potentials can be generated from a procyclic group and answer arising questions. As an expository paper, we discuss the connection between limit-periodic potentials and profinite groups as completely as possible and review some recent results on Schrödinger operators obtained in this context.
\end{abstract}

Key words: profinite group, limit-periodic potential, Schrödinger operator AMS subject classification: 47B36, 22D12, 81Q10

\section{Introduction}

This expository paper is motivated by recent research on limit-periodic Schrödinger operators $[1,4,5,6]$. In [1], Avila gave a new way to treat limit-periodic potentials by regarding limitperiodic potentials as generated by continuous sampling function along the orbits of a minimal translation of a Cantor group, allowing one to separate the base dynamics and the sampling function. Damanik and I discussed this connection in $[4,5,6]$. However, those discussions weren't very comprehensive, since the goals of these works (including Avila's [1]) were to study spectral properties of the associated Schrödinger operators, but not the connection itself. There are still some questions waiting to be clarified.

\footnotetext{
*E-mail: zheng.gan@ rice.edu
} 
By that definition, Cantor groups belong to a larger class of groups, profinite groups, that will help us to understand Cantor groups better (we shall see that a Cantor group, that admits a minimal translation, is a procyclic group). The main tasks of this paper are: (1) classification of the hulls of different limit-periodic potentials; (2) characterization of the hull of a limit-periodic potential. As an expository paper, we will cover related questions with the goal to provide a reference for future study in limit-periodic Schrödinger operators and other related topics.

\section{Classification of the Hulls of Limit-Periodic Potentials}

Definition 1. Given a topological group $\Omega$, a map $T: \Omega \rightarrow \Omega$ is called a translation if $T(\omega)=$ $\omega \cdot \omega_{0}$ for some $\omega_{0} \in \Omega$, and T is called minimal if the orbit $\left\{T^{n}(\omega): n \in \mathbb{Z}\right\}$ of every $\omega \in \Omega$ is dense in $\Omega$.

Let $\sigma$ be the left shift operator on $\ell^{\infty}(\mathbb{Z})$, that is, $(\sigma(d))_{n}=d_{n+1}$ for every $d \in \ell^{\infty}(\mathbb{Z})$. Let $\operatorname{orb}(d)=\left\{\sigma^{k}(d): k \in \mathbb{Z}\right\}$, and let hull $(d)$ be the closure of orb $(d)$ in $\ell^{\infty}(\mathbb{Z})$.

Definition 2. A potential $p \in \ell^{\infty}(\mathbb{Z})$ is called periodic if $\operatorname{orb}(p)$ is finite, and $p$ is called limitperiodic if it belongs to the closure of the set of periodic potentials.

For a limit-periodic potential $d \in \ell^{\infty}(\mathbb{Z})$, it is easy to see that every $d^{\prime} \in \operatorname{hull}(d)$ is still limitperiodic. We also have

Proposition 3. [1, Lemma 2.1] If $d$ is a limit-periodic potential, then hull $(d)$ is compact and has a unique topological group structure with identity $\sigma^{0}(d)=d$ such that

$$
\phi: \mathbb{Z} \longrightarrow \operatorname{hull}(d), \quad k \longrightarrow \sigma^{k}(d)
$$

is a homomorphism. Also, the group structure is Abelian and there exist arbitrarily small compact open neighborhoods of $d$ in hull $(d)$ that are finite index subgroups.

The last statement in Proposition 3 tells us that hull $(d)$ is totally disconnected. The translation $T: \operatorname{hull}(d) \rightarrow \operatorname{hull}(d), \omega \rightarrow \omega \cdot \sigma(d)=\sigma(\omega)$ is minimal, since $\left\{\sigma^{k}(d): k \in \mathbb{Z}\right\}$ is dense in $\operatorname{hull}(d)$.

Let us denote hull $(d)$ by $\Omega_{d}$. The dual group, $\hat{\Omega}_{d}$, of characters on $\Omega_{d}$ is naturally a topological subgroup of the circle group $C$, the multiplicative group of all complex numbers of absolute value 1 , since the dual group of $\mathbb{Z}$ is $C$. By taking inverse image under the map $\mathbb{R} \rightarrow C, \alpha \rightarrow e^{i \alpha}$, we obtain a subgroup $F_{d}$ of $\mathbb{R}$, called the frequency module of $d . F_{d}$ is countable since $\Omega_{d}$ has a countable dense subset orb $(d)$, and it is a $\mathbb{Z}$-module.

Proposition 4. [2, Theorem A.1.1] The frequency module $F_{d}$, is the $\mathbb{Z}$-module generated by

$$
\left\{\alpha: \lim _{n \rightarrow \infty} \frac{1}{2 n} \sum_{k=-n}^{n} d(k) e^{-i k \alpha} \neq 0, \alpha \in \mathbb{R}\right\} .
$$


Remark 5. Appendix 1 of [2] dealt with continuous Schrödinger operators. However, all the results from there can be directly applied to discrete Schrödinger operators as well, since the proofs in Appendix 1 of [2] don't require any specific property only successful in the continuum case.

Proposition 6. [2, Theorem A.1.3] $d \in \ell^{\infty}(\mathbb{Z})$ is limit-periodic if and only if $F_{d}$ has the property that any $\alpha, \beta \in F_{d}$ have a common divisor in $F_{d}$.

Through the Peter-Weyl theorem, we have

Proposition 7. [2, Corollary A.1.5] If $d \in \ell^{\infty}(\mathbb{Z})$ is limit-periodic, then there exists a positive integer set $S_{d}=\left\{n_{j}\right\}$ satisfying $n_{j} \mid n_{j+1}$ such that

$$
d(k)=\sum_{j=1}^{\infty} p_{j}(k),
$$

where $p_{j} \in \ell^{\infty}(\mathbb{Z})$ are $n_{j}$-periodic. This convergence is uniform.

Remark 8. Given any positive integer set $S_{d}=\left\{n_{j}\right\}$ satisfying $n_{j} \mid n_{j+1}$, let $p_{j}(k)=t / n_{j}^{3}, t=$ $k\left(\bmod n_{j}\right)$. Then the potential $d \in \ell^{\infty}(\mathbb{Z})$, defined by $d(k)=\sum_{j=1}^{\infty} p_{j}(k)$, is limit-periodic.

Given a limit-periodic potential $d \in \ell^{\infty}(\mathbb{Z})$ which is not periodic, $F_{d}$ is countable and infinitely generated (if it is finitely generated, $d$ is quasi-periodic or periodic; see Appendix 1 of [2]). Denote the set of generators of $F_{d}$ by $G_{d}=\left\{2 \pi \alpha_{j}\right\}$. By Proposition 6, $2 \pi \alpha_{1}$ and $2 \pi \alpha_{2}$ have a common divisor. By Proposition 4,

$$
a=\lim _{n \rightarrow \infty} \frac{1}{2 n} \sum_{k=-n}^{n} d(k) e^{-i 2 k \pi \alpha_{1}}
$$

and

$$
b=\lim _{n \rightarrow \infty} \frac{1}{2 n} \sum_{k=-n}^{n} d(k) e^{-i 2 k \pi \alpha_{2}}
$$

are both non-zero. Choose a periodic potential $p \in \ell^{\infty}(\mathbb{Z})$ with

$$
\|p-d\|_{\infty} \leq \frac{1}{2} \min (|a|,|b|)
$$

It follows that

$$
\lim _{n \rightarrow \infty} \frac{1}{2 n} \sum_{k=-n}^{n} p(k) e^{-i 2 k \pi \gamma} \neq 0
$$

for $\gamma=\alpha_{1}, \alpha_{2}$. So $\frac{2 \pi}{h}$ divides both $2 \pi \alpha_{1}$ and $2 \pi \alpha_{2}$ where $h$ is the period ${ }^{\dagger}$ of $p$ (note that the frequency module of an $h$-periodic potential is finitely generated, and there is a common divisor $\frac{2 \pi}{h}$ of the generators; see Appendix 1 of [2]). So there exists $n_{1} \in \mathbb{Z}^{+}$such that the greatest

\footnotetext{
${ }^{\dagger}$ In this paper, the period of a periodic potential is considered as the minimal period.
} 
common divisor of $2 \pi \alpha_{1}$ and $2 \pi \alpha_{2}$ is $\frac{2 \pi}{n_{1}}$, and the $\mathbb{Z}$-module generated by $\left\{2 \pi \alpha_{1}, 2 \pi \alpha_{2}\right\}$ is the $\mathbb{Z}$-module generated by $\left\{\frac{2 \pi}{n_{1}}\right\}$. Similarly, for $\alpha_{1}, \alpha_{2}$ and $\alpha_{3}$ there exists a positive integer $n_{2}$ such that the $\mathbb{Z}$-module generated by $\left\{2 \pi \alpha_{1}, 2 \pi \alpha_{2}, 2 \pi \alpha_{3}\right\}$ is the $\mathbb{Z}$-module generated by $\left\{\frac{2 \pi}{n_{2}}\right\}$. Clearly, $n_{1} \mid n_{2}$. By induction, we can find an infinite positive integer set

$$
S_{d}=\left\{n_{j}\right\} \subset \mathbb{Z}^{+}
$$

such that $n_{j} \mid n_{j+1}$ and $F_{d}$ is generated by $G_{d}=\left\{\frac{2 \pi}{n_{j}}: n_{j} \in S_{d}\right\}$.

We call $S_{d}$ a frequency integer set of $d$. In general, we say that $S_{d}$ is a frequency integer set of $d$ if one can obtain the frequency module $F_{d}$ from $S_{d}$. When $d$ is limit-periodic, for any $n, m \in S_{d}$, one always has $n \mid m$ or $m \mid n$. If $d(k)=\sum_{j=1}^{\infty} p_{j}(k)$, where $p_{j}$ are $n_{j}$-periodic, then it is easy to conclude that $S_{d}=\left\{n_{j}\right\}$ is a frequency integer set of $d$. Clearly, $d$ may have other frequency integer sets. By the following theorem, we shall see that there exists a unique maximal frequency integer set $M_{d}$ in the sense that every frequency integer set $S_{d}$ is contained in $M_{d}$.

Theorem 9. Given a limit-periodic potential $d \in \ell^{\infty}(\mathbb{Z})$ with an infinite frequency integer set $S_{d}$, for any limit-periodic potential $\tilde{d} \in \ell^{\infty}(\mathbb{Z})$ with a frequency integer set $S_{\tilde{d}}$, if $S_{\tilde{d}}$ is an infinite subset of $S_{d}$, then we have $\Omega_{d} \cong \Omega_{\tilde{d}}$.

Proof. Write $S_{\tilde{d}}=\left\{n_{j_{k}}\right\} \subset S_{d}=\left\{n_{j}\right\}$. Define a homomorphism by $\varphi: F_{\tilde{d}} \rightarrow F_{d}, \varphi\left(\frac{2 \pi}{n_{j_{k}}}\right)=\frac{2 \pi}{n_{j_{k}}}$. Clearly, $\varphi$ is injective since $G_{\tilde{d}} \subset G_{d}$. For any $\frac{2 \pi}{n_{t}} \notin G_{\tilde{d}}$, choose $n_{j_{k}}>n_{t}$, and then there is $t_{k}$ such that $n_{j_{k}}=t_{k} n_{t}$ (by the property of $S_{d}$ ). We have $\varphi\left(t_{k} \frac{2 \pi}{n_{j_{k}}}\right)=t_{k} \frac{2 \pi}{n_{j_{k}}}=\frac{2 \pi}{n_{t}}$, implying that $\varphi$ is also surjective. Thus, $F_{d}=F_{\tilde{d}}$. Clearly, $\hat{\Omega}_{d} \cong \hat{\Omega}_{\tilde{d}}$, since $\hat{\Omega}_{d}=\left\{e^{i \alpha}: \alpha \in F_{d}\right\}$ and $\hat{\Omega}_{\tilde{d}}=\left\{e^{i \beta}: \beta \in F_{\tilde{d}}\right\}$. By the Pontryagin duality theorem, $\Omega_{d} \cong \Omega_{\tilde{d}}$.

Theorem 9 gives us a way to find the maximal frequency integer set of $d$. If $S_{d}$ is a frequency integer set of $d$, one can add positive integers into $S_{d}$ to get $M_{d}=\left\{m_{j}\right\} \subset \mathbb{Z}^{+}$such that $m_{j+1} / m_{j}$ is a prime number. Clearly, $S_{d} \subset M_{d}$ and $M_{d}$ is still a frequency integer set of $d$. The uniqueness of $M_{d}$ follows from Theorem 9.

Theorem 10. Given limit-periodic potentials $d, \tilde{d} \in \ell^{\infty}(\mathbb{Z})$ with frequency integer sets $S_{d}$ and $S_{\tilde{d}}$ respectively, $\Omega_{d} \cong \Omega_{\tilde{d}}$ if and only iffor any $n_{i} \in S_{d}$ there exists $m_{j} \in S_{\tilde{d}}$ such that $n_{i} \mid m_{j}$, and vice versa.

Proof. If $S_{d}, S_{\tilde{d}}$ are both finite sets, $d, \tilde{d}$ are periodic, and then the statement follows readily. We assume that $S_{d}, S_{\tilde{d}}$ are infinite sets. First assume that for any $n_{i} \in S_{d}$ there exists $m_{j} \in S_{\tilde{d}}$ such that $n_{i} \mid m_{j}$ and vice versa. Then, for $n_{i_{1}} \in S_{d}$, there exists $m_{j_{1}} \in S_{\tilde{d}}$ such that $n_{i_{1}} \mid m_{j_{1}}$. Consider $m_{j_{1}} \in S_{\tilde{d}}$, and similarly, there exists $n_{i_{2}} \in S_{d}$ with $m_{j_{1}} \mid n_{i_{2}}$. By induction, we get infinite subsets $L_{d}=\left\{n_{i_{k}}\right\} \subset S_{d}$ and $L_{\tilde{d}}=\left\{m_{j_{k}}\right\} \subset S_{\tilde{d}}$ such that $H=L_{d} \cup L_{\tilde{d}}$ can be a frequency integer set of some limit-periodic potential $d^{\prime}$ (see Remark 8). By Theorem 9, we conclude that $\Omega_{d} \cong \Omega_{d^{\prime}} \cong \Omega_{\tilde{d}}$.

Conversely, assume that such a condition is not successful. Without loss of generality, suppose that for a fixed $n_{1} \in S_{d}, n_{1} \nmid m_{j}$ for any $m_{j} \in S_{\tilde{d}}$. If $\Omega_{d} \cong \Omega_{\tilde{d}}$, by the Pontryagin duality theorem, 
we have $\hat{\Omega}_{d} \cong \hat{\Omega}_{\tilde{d}}$. So there is an isomorphism $\phi: \hat{\Omega}_{d} \rightarrow \hat{\Omega}_{\tilde{d}}$ with $\phi\left(e^{2 \pi i}\right)=e^{2 \pi i}$ (the identity must be mapped to the identity). Write

$$
\phi\left(e^{\frac{2 \pi}{n_{1}} i}\right)=e^{\sum_{j=1}^{t_{1}} k_{j} \frac{2 \pi i}{m_{j}}}=e^{q_{1} \frac{2 \pi i}{m_{t_{1}}}}
$$

and we get

$$
\phi\left(e^{m_{t_{1}} \frac{2 \pi}{n_{1}} i}\right)=e^{q_{1} m_{t_{1}} \frac{2 \pi i}{m_{1}}}=e^{2 q_{1} \pi i}=1,
$$

which is impossible since $m_{t_{1}} / n_{1}$ is not an integer. Thus, $\Omega_{d} \nsucceq \Omega_{\tilde{d}}$. The proof is complete.

By Theorem 10, we conclude the following classification theorem.

Theorem 11. Given two limit-periodic potentials $d, \tilde{d} \in \ell^{\infty}(\mathbb{Z}), \Omega_{d} \cong \Omega_{\tilde{d}}$ if and only if $d$ and $\tilde{d}$ have the same maximal frequency integer set.

If $d \in \ell^{\infty}(\mathbb{Z})$ is periodic, $\operatorname{hull}(d)$ is a finite cyclic group. If $d$ is limit-periodic but not periodic, the previous theorems don't give us any information about the structure of the hull of a limitperiodic potential. We shall see that hull $(d)$ is a procyclic group later. We will also explain how to generate limit-periodic potentials from a procyclic group.

\section{Profinite Groups}

Definition 12. A group $\Omega$ is called Cantor if it is a totally disconnected compact Abelian topological group without isolated points.

Remark 13. Not every Cantor group has minimal translations. For example,

$$
\Omega=\prod_{j=0}^{\infty} \mathbb{Z}_{2},
$$

where $\mathbb{Z}_{2}$ is a cyclic 2-group, is a Cantor group with the product topology, but it has no minimal translations, since $\omega+\omega$ is the identity for any $\omega \in \Omega$.

By the definition of Cantor groups and Proposition 3, we conclude that given a limit-periodic potential $d \in \ell^{\infty}(\mathbb{Z})$ with an infinite frequency integer set (it ensures that $d$ is not periodic), hull $(d)$ is a Cantor group which has a minimal translation. A topological profinite group is by definition an inverse limit of finite topological groups. Now let's introduce the related definitions.

A directed set is a partially ordered set $I$ such that for all $i_{1}, i_{2} \in I$ there is an element $j \in I$ for which $i_{1} \leq j$ and $i_{2} \leq j$.

Definition 14. An inverse system $\left(X_{i}, \phi_{i j}\right)$ of topological groups indexed by a directed set I consists of a family $\left(X_{i} \mid i \in I\right)$ of topological groups and a family $\left(\phi_{i j}: X_{j} \rightarrow X_{i} \mid i, j \in I, i \leq j\right)$ of continuous homomorphisms such that $\phi_{i i}$ is the identity map $\operatorname{id}_{X_{i}}$ for each $i$ and $\phi_{i j} \phi_{j k}=\phi_{i k}$ whenever $i \leq j \leq k$. 
For example, let $I=\mathbb{Z}^{+}$with the usual order, let $p$ be a prime, and let $X_{i}=\mathbb{Z} / p^{i} \mathbb{Z}$ for each $i$, and for $j \geq i$ let $\phi_{i j}: X_{j} \rightarrow X_{i}$ be the map defined by

$$
\phi_{i j}\left(n+p^{j} \mathbb{Z}\right)=n+p^{i} \mathbb{Z}
$$

for each $n \in \mathbb{Z}$. Then $\left(X_{i}, \phi_{i j}\right)$ is an inverse system of finite topological groups.

Let $\left(X_{i}, \phi_{i j}\right)$ be an inverse system of topological groups and let $Y$ be a topological group. We call a family $\left(\phi_{i}: Y \rightarrow X_{i} \mid i \in I\right)$ of continuous homomorphisms compatible if $\phi_{i j} \phi_{j}=\phi_{i}$ whenever $i \leq j$; that is, each of the following diagrams

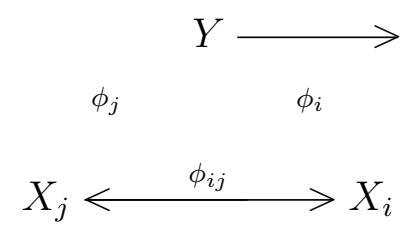

is commutative.

Definition 15. An inverse limit $\left(X, \phi_{i}\right)$ of an inverse system $\left(X_{i}, \phi_{i j}\right)$ of topological groups is a topological group together with a compatible family $\left(\phi_{i}: X \rightarrow X_{i}\right)$ of continuous homomorphisms with the following universal property: whenever $\left(\varphi_{i}: Y \rightarrow X_{i}\right)$ is a compatible family of continuous homomorphisms from a topological group $Y$, there is a unique continuous homomorphism $\varphi: Y \rightarrow X$ such that $\phi_{i} \varphi=\varphi_{i}$ for each $i$.

That is, there is a unique continuous homomorphism $\varphi$ such that each of the following diagram

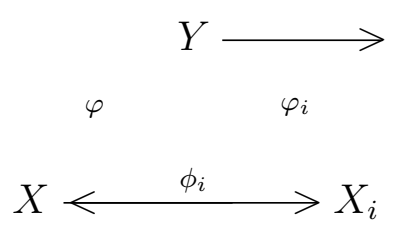

is commutative.

Proposition 16. [17, Proposition 1.1.4] Let $\left(X_{i}, \phi_{i j}\right)$ be an inverse system of topological groups, indexed by $I$.

(1). There exists an inverse limit $\left(X, \phi_{i}\right)$ of $\left(X_{i}, \phi_{i j}\right)$, for which $X$ is a topological group and the maps $\phi_{i}$ are continuous homomorphisms.

(2). If $\left(X^{(1)}, \phi_{i}^{(1)}\right)$ and $\left(X^{(2)}, \phi_{i}^{(2)}\right)$ are inverse limits of the inverse system, then there is an isomorphism $\bar{\phi}: X^{(1)} \rightarrow X^{(2)}$ such that $\phi_{i}^{(2)} \bar{\phi}=\phi_{i}^{(1)}$ for each $i$.

(3). Write $G=\prod_{i \in I} X_{i}$ with the product topology and for each $i$ write $\pi_{i}$ for the projection map from $G$ to $X_{i}$. Define

$$
X=\left\{c \in G: \phi_{i j} \pi_{j}(c)=\pi_{i}(c) \text { for all } i, j \text { with } j \geq i\right\}
$$

and $\phi_{i}=\left.\pi_{i}\right|_{X}$ for each $i$. Then $\left(X, \phi_{i}\right)$ is an inverse limit of $\left(X_{i}, \phi_{i j}\right)$. 
The result above shows that the inverse limit of an inverse system $\left(X_{i}, \phi_{i j}\right)$ exists and is unique up to isomorphism ${ }^{\ddagger}$. We also have the following important characterization of profinite groups.

Proposition 17. [17, Corollary 1.2.4] Let X be a topological group. The following are equivalent:

(1). $X$ is profinite, i.e., it is an inverse limit of an inverse system;

(2). $X$ is isomorphic to a closed subgroup of a product group of finite groups;

(3). $X$ is compact and $\bigcap\left(N \mid N \triangleleft_{O} X\right)=1$ ( $\triangleleft_{O}$ means that $N$ is open and normal);

(4). $X$ is compact and totally disconnected.

By the above proposition, we see that a Cantor group is an Abelian profinite group without isolated points.

Proposition 18. Assume that the directed set is $I=\mathbb{Z}^{+}$with the usual order. For an inverse system $\left(X_{i}, \phi_{i j}\right)_{j \geq i}$ with the inverse limit $\left(X, \phi_{i}\right)$, every non-finite sub-inverse system still has the same inverse limit $\left(X, \phi_{i_{k}}\right)$ up to isomorphism.

Proof. Consider a non-finite sub-inverse system $\left(X_{i_{k}}, \phi_{i_{k} i_{t}}\right)_{t \geq k}$. Assume that $\left(X^{\prime}, \phi_{i_{k}}^{(1)}\right)$ is the inverse limit. Obviously, $\left(X, \phi_{i_{k}}\right)$ is compatible with $\left(X_{i_{k}}, \phi_{i_{k} i_{t}}\right)_{t \geq k}$, so there is a unique homomorphism $\phi^{(1)}: X \rightarrow X^{\prime}$ such that $\phi_{i_{k}}=\phi_{i_{k}}^{(1)} \phi^{(1)}$.

For any $X_{q}$ not in the sub-inverse system, choose $i_{k}>q$. We have that $\phi_{q i_{k}}: X_{i_{k}} \rightarrow X_{q}$ and $\phi_{q}^{(1)}=\phi_{q i_{k}} \phi_{i_{k}}^{(1)}: X^{\prime} \rightarrow X_{q}$ are homomorphisms. We will prove that $\left(X^{\prime}, \phi_{i}^{(1)}\right)$ is compatible with $\left(X_{i}, \phi_{i j}\right)_{j \geq i}$, for which it suffices to show that the following diagram:

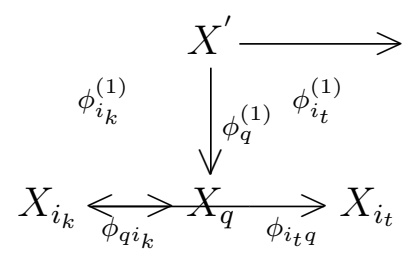

is commutative. The left half of the above diagram follows from the definition of $\phi_{q}^{(1)}$. The right half follows from $\phi_{i_{t}}^{(1)}=\phi_{i_{t} q} \phi_{q i_{k}} \phi_{i_{k}}^{(1)}=\phi_{i_{t} q} \phi_{q}^{(1)}$. So $\left(X^{\prime}, \phi_{i}^{(1)}\right)$ is compatible with $\left(X_{i}, \phi_{i j}\right)_{j \geq i}$ and there is a unique homomorphism $\phi^{(2)}: X^{\prime} \rightarrow X$ such that $\phi_{i}^{(1)}=\phi_{i} \phi^{(2)}$.

By the universal property for $\left(X^{\prime}, \phi_{i_{k}}^{(1)}\right)$, there is only one map $F: X^{\prime} \rightarrow X^{\prime}$ with the property that $\phi_{i_{k}}^{(1)} F=\phi_{i_{k}}^{(1)}$ for each $i$. However, both $\phi^{(1)} \phi^{(2)}$ and $\mathrm{id}_{X^{\prime}}$ have this property, so $\phi^{(1)} \phi^{(2)}=\mathrm{id}_{X^{\prime}}$. Similarly, for $\phi^{(2)} \phi^{(1)}: X \rightarrow X$ we have $\phi_{i_{k}} \phi^{(2)} \phi^{(1)}=\phi_{i_{k}}$. For any $\phi_{j}, \phi_{j}=\phi_{j i_{k}} \phi_{i_{k}}$ when $i_{k}>j$, so we have $\phi_{j} \phi^{(2)} \phi^{(1)}=\phi_{j i_{k}} \phi_{i_{k}} \phi^{(2)} \phi^{(1)}=\phi_{j i_{k}} \phi_{i_{k}}=\phi_{j}$, which implies $\phi^{(2)} \phi^{(1)}=\mathrm{id}{ }_{X}$. Thus, $\phi^{(1)}: X \rightarrow X^{\prime}$ is an isomorphism.

Proposition 19. [17, Proposition 1.1.7] Let G be a compact Hausdorff totally disconnected space. Then $G$ is the inverse limit of its discrete quotient spaces.

$\ddagger$ We talk about topological groups throughout the paper, for which isomorphism means group isomorphism and topological space homeomorphism, i.e., group isomorphism in addition should be continuous. 
We interpret a class in the usual sense that it is closed with respect to taking isomorphic images. Let $\mathcal{C}$ be some class of finite groups. We call a group $F$ a $\mathcal{C}$-group if $F \in \mathcal{C}$, and $G$ is called a pro- $\mathcal{C}$ group if it is an inverse limit of $\mathcal{C}$-groups. We say that $\mathcal{C}$ is closed for quotients (resp. subgroups) if every quotient group (resp. subgroup) of a $\mathcal{C}$-group is also a $\mathcal{C}$-group. Similarly, we say that $\mathcal{C}$ is closed for direct products if $F_{1} \times F_{2} \in \mathcal{C}$ whenever $F_{1} \in \mathcal{C}$ and $F_{2} \in \mathcal{C}$. For example, for the class of finite $p$-groups where $p$ is a fixed prime, an inverse limit of finite $p$-groups is called a pro- $p$ group; for the class of finite cyclic groups, an inverse limit of finite cyclic groups is called a procyclic group.

The next result describes how a given profinite group, its subgroups and quotient groups, can be represented explicitly as inverse limits.

Proposition 20. [17, Theorem 1.2.5] (1). Let G be a profinite group. If I is a filter base of closed normal subgroups of $G$ such that $\bigcap(N \mid N \in I)=1$, then

$$
G \cong \lim _{N \in I} G / N
$$

Moreover

$$
H \cong \lim _{N \in I} H /(H \cap N)
$$

for each closed subgroup $H$, and

$$
G / K \cong \lim G \in I / K N
$$

for each closed normal subgroup $K$.

(2). If $\mathcal{C}$ is a class of finite groups which is closed for subgroups and direct products, then closed subgroups, direct products and inverse limits of pro- $\mathcal{C}$ groups are pro-C groups. If in addition $\mathcal{C}$ is closed for quotients, then quotient groups of pro-C groups by closed normal subgroups are pro-C groups.

\section{More Results about Limit-periodic Potentials}

\subsection{Generate limit-periodic potentials from a procyclic group.}

We shall see how to generate limit-periodic potentials from a procyclic group, that is, a Cantor group which admits a minimal translation.

Proposition 21. [1, Lemma 2.2.] Given a Cantor group $\Omega$ and a minimal translation $T$, for each $f \in C(\Omega, \mathbb{R})$, define $F: \Omega \rightarrow \ell^{\infty}(\mathbb{Z}), F(\omega)=\left(f\left(T^{n}(\omega)\right)\right)_{n \in \mathbb{Z}}$. Then we have that $F(\omega)$ is limit-periodic and $F(\Omega)=\operatorname{hull}(F(\omega))$ for every $\omega \in \Omega$. 
By Proposition 3, we know that hull $(F(e)$ ) (e is the identity of $\Omega$ ) is a finite cyclic group or it is a Cantor group with the unique group structure: $\sigma^{0}(F(e))=F(e)$ is the identity element, and $\sigma^{i}(F(e)) \cdot \sigma^{j}(F(e))=\sigma^{i+j}(F(e))$. The translation $T$ defined by $T\left(\sigma^{i}(F(e))\right)=\sigma^{i+1}(F(e))$ is minimal.

Since $C(\Omega, \mathbb{R})$ can generate a class of limit-periodic potentials, we will get certain class of topological groups by taking the hulls of these limit-periodic potentials. The group in this class is a Cantor group or a finite cyclic group. What is the relation between this class of topological groups and the original Cantor group $\Omega$ ? To answer this question, we need two lemmas first. (Note that we will adopt the notation $F(e)$ throughout this section, that is, $F(e)=\left(f\left(T^{n}(e)\right)\right)_{n \in \mathbb{Z}}$ as in Proposition 21.)

Lemma 22. For any $f \in C(\Omega, \mathbb{R})$, hull $(F(e))$ is a quotient group of $\Omega$.

Proof. Define $\phi$ by $\phi: \Omega \longrightarrow \operatorname{hull}(F(e)), \phi(\omega)=F(\omega)$. It is not hard to see that the group structure of hull $(F(e))$ is like $F\left(\omega_{1}\right) \cdot F\left(\omega_{2}\right)=F\left(\omega_{1} \cdot \omega_{2}\right)$, since $F\left(T^{m}(e)\right)=\sigma^{m}(F(e))$. It follows that $\phi$ is a group homomorphism. By Proposition 21, $\phi$ is surjective. The continuity of $\phi$ follows from compactness of $\Omega$ and continuity of $f$. So we have

$$
\operatorname{hull}(F(e)) \cong \Omega / \operatorname{ker}(\phi),
$$

implying the lemma.

Lemma 23. There exists an $f \in C(\Omega, \mathbb{R})$ such that hull $(F(e)) \cong \Omega$.

Proof. By Lemma 22, it suffices to prove that there exists some $f \in C(\Omega, \mathbb{R})$ such that $\operatorname{ker}(\phi)=$ $\{e\}$. Clearly, a Cantor group is metrizable (recall that any separable compact space is metrizable). Introduce a metric on $\Omega$ compatible with the topology. Define a function $f: \Omega \rightarrow \mathbb{R}$ by $f(\omega)=$ $\operatorname{dist}(e, \omega)$. Clearly, $f$ is continuous, so there is a corresponding $F$ (defined as in Proposition 21) : $\Omega \rightarrow \ell^{\infty}(\mathbb{Z})$ such that hull $(F(e))$ is a quotient group of $\Omega$. Consider $\phi: \Omega \longrightarrow \operatorname{hull}(F(e)), \phi(\omega)=$ $F(\omega)$. If $F(\omega)=F(e)$, then $f(\omega)=f(e)$, that is, $\operatorname{dist}(e, \omega)=\operatorname{dist}(e, e)=0$, implying $\omega=e$ and $\operatorname{ker}(\phi)=\{e\}$.

Theorem 24. Given a Cantor group $\Omega$ and a minimal translation $T$, for each $f \in C(\Omega, \mathbb{R})$, hull $(F(e))$ is a Cantor group or a finite cyclic group, and $C(\Omega, \mathbb{R})$ can generate a class of topological groups. Then there is a one-to-one correspondence between this class of topological groups and quotient groups of $\Omega$.

Proof. By Lemma 22, we know that groups in this class are quotient groups of $\Omega$. Let's show the converse direction. By the definition, a quotient group of a Cantor group is still Cantor or finitely cyclic. Given a quotient group $\Omega_{0}$ and a quotient homomorphism $q: \Omega \rightarrow \Omega_{0}$, we claim that $T$ will induce a minimal translation $T_{0}$ on $\Omega_{0}$ such that $T_{0}([\omega])=q(T(\omega))$, $[\omega] \in \Omega_{0}$. For writing convenience, we assume that the group operation is addition and $T(\omega)=\omega+\omega_{1}$. If $[\omega]=\left[\omega^{\prime}\right]$, then $T_{0}([\omega])=q(T(\omega))=q\left(\omega_{1}+\omega\right)=q\left(\omega_{1}\right)+q(\omega)=\left[\omega_{1}\right]+[\omega]=\left[\omega_{1}\right]+\left[\omega^{\prime}\right]=T_{0}\left(\left[\omega^{\prime}\right]\right)$, which gives that $T_{0}$ is a translation by $\left[\omega_{1}\right]$. That $T_{0}$ is minimal follows from the fact that $T$ is minimal and $q$ is continuous. By Lemma 23, we know that for $\Omega_{0}$ and $T_{0}$ there exists some $f_{0} \in C\left(\Omega_{0}, \mathbb{R}\right)$ 
such that $\operatorname{hull}\left(\left(f_{0}\left(T_{0}^{n}([e])\right)\right)_{n \in \mathbb{Z}}\right) \cong \Omega_{0}$. Let $f=f_{0} \circ q$. Clearly, $f \in C(\Omega, \mathbb{R})$ and the following diagram

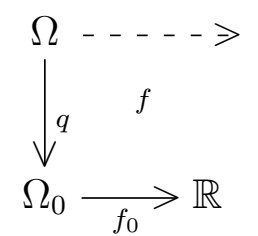

is commutative. So we have $\operatorname{hull}(F(e)) \cong \operatorname{hull}\left(\left(f_{0}\left(T_{0}^{n}([e])\right)\right)_{n \in \mathbb{Z}}\right) \cong \Omega_{0}$. The proof is complete.

We also have

Theorem 25. Given a Cantor group $\Omega$ and a minimal translation $T$, for any limit-periodic potential $d \in \ell^{\infty}(\mathbb{Z})$ satisfying hull $(d) \cong \Omega$, there is an $f \in C(\Omega, \mathbb{R})$ such that $f\left(T^{n}(e)\right)=d_{n}$ for every $n \in \mathbb{Z}$.

Proof. By Lemma 23 we have $\tilde{f} \in C(\Omega, R)$ such that hull $(\tilde{F}(e)) \cong \Omega$ (note that $\tilde{F}(e)=$ $\left.\left(\tilde{f}\left(T^{n}(e)\right)\right)_{n \in \mathbb{Z}}\right)$. Since hull $(d) \cong \Omega$, we have a continuous isomorphism $h: \operatorname{hull}(\tilde{F}(e)) \rightarrow \operatorname{hull}(d)$ with $h(\tilde{F}(e))=d$.

Clearly, for $T^{n_{k}}(e) \in \Omega$ we have $h\left(\tilde{F}\left(T^{n_{k}}(e)\right)\right)=\sigma^{n_{k}}(d)$ since $\tilde{F}\left(T^{n_{k}}(e)\right)=\sigma^{n_{k}}(\tilde{F}(e))$. If $\lim _{k \rightarrow \infty} T^{n_{k}}(e)=\omega$, then $h(\tilde{F}(\omega))=\lim _{k \rightarrow \infty} \sigma^{n_{k}}(d)$, where the limit exists since $h$ and $\tilde{F}$ are both continuous. Define $f$ by $f\left(T^{n}(e)\right)=\sigma^{n}(d)_{0}=d_{n}$. We extend $f$ to the whole $\Omega$ by $f(\omega)=\lim _{k \rightarrow \infty} \sigma^{n_{k}}(d)_{0}$ if $\omega=\lim _{k \rightarrow \infty} T^{n_{k}}(e)$. By the previous analysis, $f$ is well defined and continuous. So there is an $f \in C(\Omega, \mathbb{R})$ such that $F(e)=d$, that is, $f\left(T^{n}(e)\right)=d_{n}$ for every $n \in \mathbb{Z}$.

We say that $f \in C(\Omega, \mathbb{R})$ is $p$-periodic with respect to $T$ if $f\left(T^{p}(\omega)\right)=f(\omega)$ for every $\omega \in \Omega$. Then we have

Proposition 26. [5, Proposition 2.7] Let $f \in C(\Omega, \mathbb{R})$. If $f\left(T^{p+m}\left(\omega_{0}\right)\right)=f\left(T^{m} \omega_{0}\right)$ for some $\omega_{0} \in \Omega$, some minimal translation $T: \Omega \rightarrow \Omega$ and every $m \in \mathbb{Z}$, then for every minimal translation $\tilde{T}: \Omega \rightarrow \Omega, f$ is p-periodic with respect to $\tilde{T}$.

The above proposition tells us that the periodicity of $f$ is independent of minimal translations. Next we recall from [1] how periodic functions in $C(\Omega, \mathbb{R})$ can be constructed. Given a compact open subgroup $\Omega_{0}$ with finite index (such open subgroups can be found in any neighborhood of the identity element; see Proposition 3) and $f \in C(\Omega, \mathbb{R})$, we can define a periodic $f_{\Omega_{0}} \in C(\Omega, \mathbb{R})$ by

$$
f_{\Omega_{0}}(\omega)=\int_{\Omega_{0}} f(\omega \cdot \tilde{\omega}) d \mu_{\Omega_{0}}(\tilde{\omega}),
$$

where $\mu_{\Omega_{0}}$ denotes the normalized Haar measure on $\Omega_{0}$. This shows that the set of periodic functions is dense in $C(\Omega, \mathbb{R})$ since $\Omega_{0}$ can be arbitrarily small. Moreover, there exists a decreasing sequence of Cantor subgroups $\Omega_{k}$ with finite index $n_{k}$ such that $\bigcap \Omega_{k}=\{e\}$ (we will see this point explicitly in the next subsection). Let $P_{k}$ be the set of functions defined on $\Omega / \Omega_{k}$, that is, the elements in $P_{k}$ are $n_{k}$-periodic potentials. Denote by $P$ the set of all periodic functions. Then, we have $P_{k} \subset P_{k+1}$ and $P=\bigcup P_{k}$. 


\subsection{Characterization of the hull of a limit-periodic potential}

Given a limit-periodic potential $d \in \ell^{\infty}(\mathbb{Z})$ with an infinite frequency integer set $S_{d}=\left\{n_{j}\right\}$, we see that hull $(d)$ is a Cantor group admitting a minimal translation. Consider the directed set $I=\mathbb{Z}^{+}$with the usual order. This gives rise to an inverse system $\left(\mathbb{Z}_{n_{i}}, \pi_{i j}\right)_{j \geq i}$, where $\mathbb{Z}_{n_{j}}$ are $n_{j}$-cyclic groups with the discrete topology and $\pi_{i j}$ is a homomorphism defined by $\pi_{i j}\left(k+n_{j} \mathbb{Z}\right)=$ $k+n_{i} \mathbb{Z}, k \in \mathbb{Z}$.

We endow $\mathbb{Z}_{n_{j}}$ with a discrete metric defined by $\operatorname{dist}_{j}\left(a_{1}, a_{2}\right)=0$ when $a_{1}=a_{2}$ and $\operatorname{dist}_{j}\left(a_{1}, a_{2}\right)=1$ when $a_{1} \neq a_{2}$. Consider the product group

$$
A=\prod_{j=1}^{\infty} \mathbb{Z}_{n_{j}}
$$

of which the topology is the product topology. We endow $A$ with a metric defined by

$$
\operatorname{dist}(x, y)=\sum_{j=1}^{\infty} \frac{1}{2^{j}} \frac{\operatorname{dist}_{j}\left(x_{j}, y_{j}\right)}{1+\operatorname{dist}_{j}\left(x_{j}, y_{j}\right)}, \quad x, y \in A,
$$

which is compatible with the product topology.

Let $E=(1,1, \cdots, 1, \cdots) \in A$, and consider the closed subgroup

$$
\bar{B}=\overline{\{n E=(n, n, \cdots, n, \cdots) \in A: n \in \mathbb{Z}\}} .
$$

Obviously, $\bar{B}$ is a Cantor group with a minimal translation $T(x)=x+E$, and $\overrightarrow{0} \in \bar{B}$ is the identity element. Let

$$
\tilde{d}_{k}=\operatorname{dist}(k E, \overrightarrow{0})=\sum_{j=1}^{\infty} \frac{1}{2^{j}} \frac{\operatorname{dist}_{j}(k, 0)}{1+\operatorname{dist}_{j}(k, 0)} .
$$

By the proof of Lemma 23, we know that $\tilde{d}=\left(\tilde{d}_{k}\right)_{k \in \mathbb{Z}}$ is limit-periodic and

$$
\operatorname{hull}(\tilde{d}) \cong \bar{B} \text {. }
$$

Let $p_{j}(k)=\frac{1}{2^{j}} \frac{\operatorname{dist}_{j}(k, 0)}{1+\operatorname{dist}_{j}(k, 0)}$. Then we have

$$
\tilde{d}(k)=\sum_{j=1}^{\infty} p_{j}(k)
$$

which tells us that one of $\tilde{d}$ 's frequency integer sets is $S_{d}=\left\{n_{j}\right\}$. By Theorem 9, we conclude that

$$
\operatorname{hull}(d) \cong \operatorname{hull}(\tilde{d}) \cong \bar{B}
$$

Let $\bar{B}_{k}=\overline{\left\{n n_{k} E: n \in \mathbb{Z}\right\}} \subset \bar{B}$, and it is easy to see that there exists a decreasing sequence of Cantor subgroups $\bar{B}_{k}$ with the index $n_{k}$ and $\bigcap \bar{B}_{k}=\{\overrightarrow{0}\}$. 
Proposition 27. $b=(k, k, \cdots, k, \cdots)$ is a generator in $\bar{B}$, that is, $\{n b: n \in \mathbb{Z}\}$ is dense in $\bar{B}$, if and only if, for any $n_{j}, k$ and $n_{j}$ have no common divisors.

Proof. If there exists some $n_{t}$ such that $\left(k, n_{t}\right)=k_{t}>1$, then $k$ cannot be a generator for $Z_{n_{t}}$, i.e. $n k \neq 1\left(\bmod n_{t}\right)$ for any $n \in \mathbb{Z}$, and then

$$
\inf _{n \in \mathbb{Z}}\|n b-E\| \geq \frac{1}{2^{t}} \frac{\operatorname{dist}_{t}(n k, 1)}{1+\operatorname{dist}_{t}(n k, 1)}=\frac{1}{2^{t+1}},
$$

where $E=(1,1, \cdots, 1, \cdots)$. So $b$ is not a generator.

Conversely, if for any $n_{j}, k$ and $n_{j}$ have no common divisors, i.e. $\left(k, n_{j}\right)=1$, we will show that there exists a positive integer sequence $\left\{q_{j}\right\}_{j \in \mathbb{Z}^{+}}$such that $\lim _{j \rightarrow \infty} q_{j} b=E$ in the norm sense. Since $\left(k, n_{1}\right)=1$, there exists some positive integer $q_{1}$ such that $q_{1} k=1\left(\bmod n_{1}\right)$. Similarly, since $\left(k, n_{2}\right)=1$, we have $q_{2} k=1\left(\bmod n_{2}\right)$. Since $n_{1} \mid n_{2}$, we still have $q_{2} k=1\left(\bmod n_{1}\right)$. By induction, we get a sequence $\left\{q_{j}\right\}_{j \in \mathbb{Z}^{+}}$such that $q_{j} k=1\left(\bmod n_{i}\right)$ when $i \leq j$. It is easy to see that $\lim _{j \rightarrow \infty} q_{j} b=E$ in the norm sense, and so $b$ is a generator.

Proposition 28. Let $T: \bar{B} \rightarrow \bar{B}, x \rightarrow b+x$. $T$ is minimal if and only if $b$ is a generator.

Proof. If $b$ is not a generator, clearly $T$ is not minimal. On the other hand, if $b$ is a generator, for each $x \in \bar{B}$, there exists a corresponding sequence $\left\{q_{j}\right\}_{j \in \mathbb{Z}^{+}}$such that $\lim _{j \rightarrow \infty} q_{j} b=E-x(E$ is the same as before), and so $\lim _{j \rightarrow \infty}\left(q_{j} b+x\right)=E$, which implies $\lim _{j \rightarrow \infty} T^{q_{j}}(x)=E$. It follows that $T$ is minimal.

Furthermore, since the following diagram

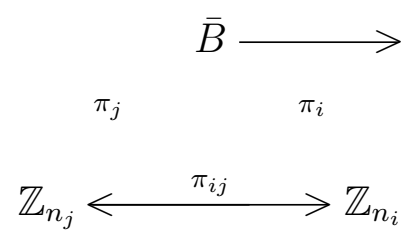

is commutative where $\pi_{i}$ is the $i$-th coordinate projection, $\left(\bar{B}, \pi_{i}\right)$ is compatible with this inverse system $\left(\mathbb{Z}_{n_{i}}, \pi_{i j}\right)_{j \geq i}$. Proposition 16 ensures that $\left(\bar{B}, \pi_{i}\right)$ is also the inverse limit of this system (see the statement (3) of Proposition 16). As already introduced, we call $\bar{B}$ a procyclic group, that is, an inverse limit of finite cyclic groups. Equivalently, a procyclic group is a profinite group that can be generated by one element. Thus, we have proved that a Cantor group with a minimal translation is a procyclic group. Also, equivalently, we have the following.

Theorem 29. For any limit-periodic potential $d \in \ell^{\infty}(\mathbb{Z})$, hull $(d)$ is a procyclic group.

By the above analysis and Proposition 18, Theorem 9 follows directly. There is also a classification theorem about procyclic groups, which is essentially the same as Theorem 10. Let $n=$ $\prod_{p} p^{n(p)}$ be a supernatural number where $p$ goes through all the primes with $0 \leq n(p) \leq \infty$ (one can consider this expression as the generalized prime factorization, and we call it supernatural since it is an extension of natural numbers to infinities that differ by different factorizations). 
Theorem 30. [15, Theorem 2.7.2] There exists a unique procyclic group G of order $n$ up to isomorphism.

Remark 31. (1). If $n=p^{n(p)}$, where $p$ is a prime and $n(p)=\infty$, then the associated procyclic group is the group of p-adic integers (see p.26 of [15]).

(2). Given a limit-periodic potential $d \in \ell^{\infty}(\mathbb{Z})$, hull $(d)$ has order $n$. If $S_{d}=\left\{n_{j}\right\}$ is a frequency integer set of $d$, then one must have $\lim _{j \rightarrow \infty} n_{j}=n$ (here "=" means that they have the same generalized prime factorization).

At the end of this section, we discuss quotient groups of a procyclic group. The class of cyclic groups is closed with respect to quotients, that is, a quotient group of a cyclic group is still cyclic. By Proposition 20, we know that a quotient group of a procyclic group is still procyclic. We have

Proposition 32. Given a procyclic group $G$ with order $n=\prod_{j \in \mathbb{Z}^{+}} p_{j}^{r_{j}}$ where $p_{j}$ are primes with $0<r_{j} \leq \infty$, quotient groups of $G$ are procyclic groups with order $m=\prod_{j \in \mathbb{Z}^{+}} p_{j}^{a_{j}}$ where $0 \leq a_{j} \leq r_{j}$, and vice versa.

Proof. Obviously, $G \cong \lim _{\leftarrow k} Z_{n_{k}}$, where $n_{k} \mid n_{k+1}$ and $\lim _{k \rightarrow \infty} n_{k}=n$. Write $\bar{B}=\overline{\{n E: n \in \mathbb{Z}\}}$, where $E=(1,1, \cdots, 1, \cdots) \in \prod_{k} Z_{n_{k}}$. Then, $\bar{B} \cong \lim _{\leftarrow k} Z_{n_{k}}$. It is sufficient to consider $\bar{B}$.

Write $\bar{B}_{k}=\overline{\left\{n n_{k} E: n \in \mathbb{Z}\right\}}$. Clearly, $\left\{\bar{B}_{k}\right\}$ is a decreasing sequence of open (also closed) subgroups of $\bar{B}$ with index $n_{k}$ and $\bigcap \bar{B}_{k}=\{\overrightarrow{0}\}$. By Proposition 20, for every closed subgroup $N \subset \bar{B}$ (every subgroup is normal in an Abelian group), we have $\bar{B} / N \cong \lim _{\leftarrow k} \bar{B} / \bar{B}_{k} N$. Since $\bar{B} / \bar{B}_{k}$ is an $n_{k}$-cyclic group, $\bar{B} / \bar{B}_{k} N$ is an $m_{k}$-cyclic group with $m_{k} \mid n_{k} . B / N$ is a procyclic group with order $m=\lim _{k \rightarrow \infty} m_{k}$. Since $m_{k} \mid n_{k}$, it follows that $m=\prod_{j \in \mathbb{Z}^{+}} p_{j}^{a_{j}}$ where $0 \leq a_{j} \leq r_{j}$.

Conversely, if $m=\prod_{j \in \mathbb{Z}^{+}} p_{j}^{a_{j}}$ where $0 \leq a_{j} \leq r_{j}$, obviously there exist $m_{k}$ such that $m_{k} \mid n_{k}$ and $\lim _{k \rightarrow \infty} m_{k}=m$. Let $\tilde{B}=\lim _{\leftarrow k} Z_{m_{k}}$, and define $\phi: \bar{B} \rightarrow \tilde{B}, \phi(E)=\tilde{E}$, where $\tilde{E}=$ $(1,1, \cdots, 1, \cdots) \in \prod_{k} Z_{m_{k}}$. Metrics on $\tilde{B}$ and $\bar{B}$ are the metric like (4.1). It is easy to see that $\phi$ is a continuous surjective homomorphism. So $\tilde{B}$ is a quotient group of $\bar{B}$.

Remark 33. By the above proposition, it is easy to see that there exists a universal procyclic group with order $n=\prod_{p} p^{\infty}$, where p goes though all the primes, in the sense that any procyclic group is a quotient group of this group.

\section{Applications in the Schrödinger Operators}

In this section, we will review some recent results on the limit-periodic Schrödinger operators obtained in this context. First, let us describe the model. We consider Schrödinger operators $H_{f, T}^{\omega}$ acting in $\ell^{2}(\mathbb{Z})$ with dynamically defined potentials $V_{f, T}^{\omega}$ given by

$$
\left[H_{f, T}^{\omega} u\right](n)=u(n+1)+u(n-1)+V_{f, T}^{\omega}(n) u(n),
$$


where

$$
V_{f, T}^{\omega}(n)=f\left(T^{n}(\omega)\right), \quad \omega \in \Omega, n \in \mathbb{Z}
$$

with a homeomorphism $T$ of a compact space $\Omega$ and a continuous sampling function $f: \Omega \rightarrow \mathbb{R}$.

We have seen that once $\Omega$ is a Cantor group and $T$ a minimal translation, $V_{f, T}^{\omega}(n)=f\left(T^{n}(\omega)\right)$ is a limit-periodic potential. Clearly, $T$ is ergodic with respect to $\mu$, the normalized Haar measure on $\Omega$. The ability to fix the base dynamics and independently vary the sampling functions is very useful in constructing examples of Schrödinger operators with desired spectral features.

Theorem 34. [1, Theorem 1.1] Suppose $\Omega$ is a Cantor group and $T$ a minimal translation. For a dense set of $f \in C(\Omega, \mathbb{R})$ and every $\lambda \neq 0$, the spectrum of $H_{\lambda f, T}^{\omega}$ has zero Lebesgue measure, and the Lyapunov exponent is a continuous positive function of the energy.

By the above theorem, Avila gave first examples of ergodic potentials with a spectrum of zero Lebesgue measure such that the Lyapunov exponent is positive throughout the spectrum. This answers a question raised by Simon (Conjecture 8.1 of [16]).

Theorem 35. [4, Theorem 1.1] Suppose $\Omega$ is a Cantor group and $T$ a minimal translation. We have

(1) For a dense set of $f \in C(\Omega, \mathbb{R})$ and $\lambda \neq 0$, the spectrum of $H_{\lambda f, T}^{\omega}$ is a Cantor set of positive Lebesgue measure and purely absolutely continuous.

(2) For a dense $G_{\delta}$-set of $f \in C(\Omega, \mathbb{R})$, every $\lambda \neq 0$ and every $\omega \in \Omega$, the spectrum of $H_{\lambda f, T}^{\omega}$ is a Cantor set of zero Lebesgue measure and purely singular continuous.

The proof of the above theorem shows that the Lyapunov exponent vanishes throughout the spectrum. This gives spectral information about the limit-periodic potentials in the regime of zero Lyapunov exponents.

Definition 36. A bounded map $V: \mathbb{Z} \rightarrow \mathbb{R}$ is called a Gordon potential if there exist positive integers $q_{j} \rightarrow \infty$ such that

$$
\max _{1 \leq n \leq q_{j}}\left|V(n)-V\left(n \pm q_{j}\right)\right| \leq j^{-q_{j}}
$$

for every $j \geq 1$.

Theorem 37. [5, Theorem 1.3] Suppose $\Omega$ is a Cantor group and $T$ a minimal translation. Then there exists a dense set $\mathcal{F} \subset C(\Omega, \mathbb{R})$ such that for every $f \in \mathcal{F}$, every $\lambda \neq 0$ and every $\omega \in \Omega$, the following statements hold true: the spectrum of $H_{\lambda f, T}^{\omega}$ has zero Hausdorff dimension, it is purely singular continuous, and $E \mapsto L(E, T, \lambda f)$ is a positive continuous function.

Theorem 38. [5, Theorem 1.5] Suppose $\Omega$ is a Cantor group and $T$ a minimal translation. Then there exists a dense set $\mathcal{F} \subset C(\Omega, \mathbb{R})$ such that for every $f \in \mathcal{F}$, every minimal translation $T: \Omega \rightarrow \Omega$, every $\omega \in \Omega$, and every $\lambda \neq 0, \lambda f\left(T^{n}(\omega)\right)$ is a Gordon potential. 
Theorem 37 and Theorem 38 are both from [5]. The Gordon lemma ensures that the Schrödinger operators with Gordon potentials have no point spectrum. The proof of Theorem 37 used Theorem 38 to conclude absence of point spectrum. Absence of a.c. spectrum follows from zero Hausdorff dimension. Theorem 37 gives first examples of limit-periodic potentials with purely singular continuous spectrum in the regime of positive Lyapunov exponents.

Also, recent work of Damanik and Gorodetski, announced in [7], focused on the weakly coupled Fibonacci Hamiltonian, that is an ergodic model not (uniformly) almost periodic. One of the theorems in [7] says that the Hausdorff dimension of the spectrum, as a function of the coupling constant, is continuous at zero. In contrast to this, Theorem 37 tells us that there are limit-periodic potentials such that continuity at zero coupling fails since the Hausdorff dimension of the spectrum is zero for all non-zero values of the coupling constant.

Definition 39. We say that a family $\left\{u_{j}\right\} \subset \ell^{2}(\mathbb{Z})$ is uniformly localized if there exist constants $r>0$, called the decay rate, and $c<\infty$ such that for every element $u_{j}$ of the family, one can find $m_{j} \in \mathbb{Z}$, called the center of localization, so that $\left|u_{j}(n)\right| \leq c e^{-r\left|n-m_{j}\right|}$ for every $n \in \mathbb{Z}$. We say that the operator $H_{\omega}$ has ULE if it has a complete set of uniformly localized eigenfunctions. ${ }^{\S}$

For any Cantor group $\Omega$ that admits a minimal translation $T$, there exists a limit-periodic potential $d$ such that hull $(d) \cong \Omega$. Write $M_{d}$ as the maximal frequency integer set of $d$. We also say that $M_{d}$ is the maximal frequency integer set of $\Omega$. Theorem 11 ensures that it is well defined.

Definition 40. Given a Cantor group that admits a minimal translation, we say that it satisfies the condition $\mathscr{A}$ if its maximal frequency integer set $M=\left\{m_{j}\right\} \subset \mathbb{Z}^{+}$has the following property: there exists some integer $m \geq 2$ such that for every $j$, we have $m_{j}<m_{j+1} \leq m_{j}^{m}$, that is, $\log m_{j+1} / \log m_{j}$ is uniformly bounded.

Theorem 41. [6, Theorem 2.5] Suppose that $\Omega$ satisfies the condition $\mathscr{A}$. Then there exists an $f \in C(\Omega, \mathbb{R})$ such that for every $\omega \in \Omega, H_{f, T}^{\omega}$ has the same pure point spectrum and ULE with $\omega$-independent constants.

The authors of [6] conclude this result by applying Pöschel' general theorem [13] to the limit-periodic potentials. Since localization is a topic that has been explored in the context of Schrödinger operators to a great extent, we would like to talk more about this result.

In the topological setting, if $T$ is minimal and $f$ continuous, the spectrum and the a.c. spectrum are independent of $\omega$; however, the point spectrum and the s.c. spectrum are in general not. If pure point spectrum is independent of $\omega$, we call it phase stable. This phenomenon is unusual since most of known models are not phase stable. For example, it is well known that pure point spectrum cannot be independent of $\omega$ in quasi-periodic Schrödinger operators [12]; for the Anderson model, one can find periodic sequences $\omega$ making the spectrum absolutely continuous. Even for the Maryland model, the Schrödinger operator (5.1) with $\Omega=S^{1}$ and $V_{\omega}(n)=\lambda \tan (\omega+2 \pi n \alpha)$, where $\alpha$ is a diophantine irrational, we only know that for every $\omega \in \Omega \backslash\left\{\frac{\pi}{2}+\mathbb{Z}+\alpha \mathbb{Z}\right\}$ and any $\lambda \neq 0$, the Schrödinger operator (5.1) has a pure point spectrum $[8,9,14]$. Theorem 41 gives the

\footnotetext{
${ }^{\S}$ Recall that a set of vectors is called complete if their span (i.e., the set of finite linear combinations of vectors from this set) is dense.
} 
first example whose spectrum is phase stable (modulo some unpublished work of Jitomirskaya, which established a similar result for a bounded non-almost periodic potential). Furthermore, the example has ULE for every $\omega \in \Omega$, which is stronger than phase stability (please refer to [11]).

There are two open questions. Is there ULE in a procyclic group of other type, i.e., a Cantor group with minimal translations that don't satisfy the condition $\mathscr{A}$ ? Given a Cantor group $\Omega$ that admits a minimal translation, does there exist a dense set of $f \in C(\Omega, \mathbb{R})$ such that the spectrum is pure point?

Theorem 42. [10, Theorem 1.2] Given any increasing continuous function $\varphi: \mathbb{R}^{+} \rightarrow \mathbb{R}^{+}$with

$$
\lim _{x \rightarrow 0} \varphi(x)=0
$$

and a constant $C_{0}>0$, there is a limit-periodic $V$ satisfying $\|V\|_{\infty} \leq C_{0}$ such that the associated integrated density of states satisfies

$$
\limsup _{E \rightarrow E_{0}} \frac{\left|k_{V}(E)-k_{V}\left(E_{0}\right)\right| \log \left(\left|E-E_{0}\right|^{-1}\right)}{\varphi\left(\left|E-E_{0}\right|\right)}=\infty
$$

for any $E_{0} \in \sigma(\Delta+V)$.

The above limit-periodic potential was also constructed through a Cantor group and a minimal translation. The authors [10] heavily use [1, Lemma 3.1, Lemma 3.2] to conclude Theorem 42. Craig and Simon in [3] showed that integrated density of states are in general log Hölder continuous. Theorem 42 tells us that continuity of integrated density of states cannot be improved for all potentials beyond log Hölder continuity. These limit-periodic potentials are dense in the space of limit-periodic potentials.

\section{Acknowledgements}

I am deeply grateful to David Damanik, who introduced me to this topic and encouraged me to write this expository paper. Also, his comments have been very helpful for completion of the paper. I also thank the referee for useful suggestions on how to improve the presentation.

\section{References}

[1] A. Avila. On the spectrum and Lyapunov exponent of limit periodic Schrödinger operators. Commun. Math. Phys., 288 (2009), 907-918.

[2] J. Avron, B. Simon. Almost periodic Schrödinger operators. I. Limit periodic potentials. Commun. Math. Phys., 82 (1981), 101-120.

[3] W. Craig, B. Simon. Subharmonicity of the Lyaponov index. Duke Math. J., 50:2 (1983), $551-560$. 
[4] D. Damanik, Z. Gan. Spectral properties of limit-periodic Schrödinger operators. To appear in to appear in Discrete Contin. Dyn. Syst. Ser. S.

[5] D. Damanik, Z. Gan. Limit-periodic Schrödinger operators in the regime of positive Lyapunov exponents. J. Funct. Anal. 258:12 (2010), 4010-4025

[6] D. Damanik, Z. Gan. Limit-periodic Schrödinger operators with uniformly localized eigenfunctions. Preprint, (arXiv:1003.1695).

[7] D. Damanik, A. Gorodetski. The spectrum of the weakly coupled Fibonacci Hamiltonian. Electron. Res. Announc. Math. Sci., 16 (2009), 23-29.

[8] A. Figotin, L. Pastur. An exactly solvable model of a multidimensional incommensurate structure. Commun. Math. Phys., 95 (1984), 401-425.

[9] S. Fishman, D. Grempel, R. Prange. Localization in a d-dimensional incommensurate structure. Phys. Rev. B, 29 (1984), 4272-4276.

[10] Z. Gan, H. Krüger. Optimality of log Hölder continuity of the integrated density of states. To appear in Math. Nachr.

[11] S. Jitomirskaya. Continuous spectrum and uniform localization for ergodic Schrödinger operators. J. Funct. Anal., 145 (1997), 312-322.

[12] S. Jitomirskaya, B. Simon. Operators with singular continuous spectrum, III. Alomost periodic Schrödinger operators. Commun. Math. Phys., 165 (1994), 201-205.

[13] J. Pöschel. Examples of discrete Schrödinger operators with pure point spectrum. Commun. Math. Phys., 88 (1983), 447-463.

[14] R. Prange, D. Grempel, S. Fishman. A solvable model of quantum motion in an incommensurate potential. Phys. Rev. B, 29 (1984), 6500-6512.

[15] L. Ribes, P. Zalesskii. Profinite Groups. Springer-Verlag, Berlin, 2000.

[16] B. Simon. Equilibrium measures and capacities in spectral theory. Inverse Probl. Imaging, 1 (2007), No. 4, 713-772.

[17] J. Wilson. Profinite Groups. Oxford University Press, New York, 1998. 